\title{
Nanoparticles in The ETEM: From Gas-Surface Interactions of Single Objects to Collective Behavior of Nanocatalysts
}

\author{
Thierry Epicier ${ }^{1}$ \\ 1. University of Lyon, INSA-Lyon, UCBL Lyon 1, MATEIS, umr5510 CNRS, Bât. Blaise Pascal, 69621 \\ Villeurbanne Cedex, France.
}

Environmental Transmission Electron Microscopy (ETEM) has received a considerable amount of interest in the last few years, both from a technology and applications points of view. In addition to closed cells, dedicated instruments allow readily to follow the evolution of nano-objects in situ in the microscope, under variable gas and temperature conditions, down to the atomic resolution (see some representative reviews cited as ref. [1)]. Since gas and temperature environments constitute the natural working conditions for catalyst systems, ETEM appears as a new efficient toolbox that chemists may employ in complement to usual spectroscopic methods aiming at characterizing the activity of catalysts in operation.

The present contribution is a review of some recent studies performed in this field, mainly on metallic and oxide nanoparticles (NPs) using the dedicated ETEM instrument installed at CLYM in Lyon, F. The microscope, an objective Cs-corrected TITAN ETEM 80-300 kV from FEI, is equipped with usual analytical devices: XMAX SDD EDX detector from Oxford Instruments, Tridiem ERS Gatan Imaging Filter. A last generation CMOS-based 4K OneView camera from Gatan allows high speed acquisitions, an advantage which will be profitable for fast environmental nano-tomography as it will be briefly presented through dedicated examples. Specific sample-holders (Wildfire S5 heating holder from DENS Solutions and Picondenter PI95 from Hysitron) compatible with the environmental mode complete the accessories. Illustrations of several experiments: (fast) HRTEM, HRSTEM, STEM-EELS, fast tomography will be reported, using all accessories on isolated or collection of supported NPs in situ under gaseous atmospheres. Two specific examples are illustrated below.

A first application concerns nano-catalysts developed for depollution of Diesel motors emissions [2]. This topic of high societal impact is severely controlled in most advanced countries through consistently updated regulations limiting the production of hazardous pollutants (such as NOx) and Particulate emission based on unburned carbon matter. Figure 1 illustrates an in situ sequence where carbon soots are consumed at $495^{\circ} \mathrm{C}$ under oxygen at the contact of zirconia-based particles.

A second study deals with ceria nanocubes exposed to different oxidizing / reducing conditions in the ETEM. Ceria (Cerium dioxide $\mathrm{CeO}_{2}$ ) is a fascinating material used in many technological fields, from catalysis to medicine or energy applications [3]. It holds its unique properties to the ability to accept and donate electrons through an easy change in the $\mathrm{Ce}$ valence, from $\mathrm{Ce}^{4+}$ in $\mathrm{CeO}_{2}$ to $\mathrm{Ce}^{3+}$ in $\mathrm{Ce}_{2} \mathrm{O}_{3}$. We will focus on the atomic arrangement of main $\{100\}$ surfaces when the cubes are observed under high vacuum (an 'aggressive' reducing condition producing a fast irradiation-induced oxygen substoichiometry, especially in TEM at $300 \mathrm{kV}$ ) or under oxidizing conditions (oxygen or $\mathrm{CO}_{2}$ ) at room and high temperature. Figure 2 demonstrates that profile imaging of cubes hanging on the edge of holes or cracks in the supporting film of the TEM grid allows the atomic mobility of $\mathrm{Ce}$ and even $\mathrm{O}$ atoms to be followed at high frequencies, owing to the high speed camera. 


\section{References:}

[1] J Jinschek, Chem. Commun., 50 (2014), p. 2696; E D Boyes, P Gai, C. R. Physique 15 (2014), p. 200; P.A. Crozier, T.W. Hansen, MRS Bull. 40 (2015), p. 38; S Takeda, Y Kuwauchi,H Yoshida, Ultramicroscopy 151 (2015), p. 178.

[2] A Serve et al, Applied Catalysis A: General 504 (2015), p. 74.

[3] C Sun, H Lia, L Chen, Energy Environ. Sci., 5 (2012), p. 8475.

[4] The author acknowledges funding from the French National Research Agency ANR (3DClean project $\left.\mathrm{n}^{\circ} 15-\mathrm{CE} 09-0009-01\right)$. Several collaborators from MATEIS ${ }^{\S}$ and IRCELYON $^{\dagger}$ (University of Lyon) and IFPen $\$$ (Solaize, F) are warmly thanked for their contributions (providing samples and problematics, discussions, participation to the observations): S. Koneti ${ }^{\S}$, Drs L. Roiban ${ }^{\S}$, C. Langlois ${ }^{\S}$, M. Bugnet ${ }^{\S}$, S. Li ${ }^{\dagger}$, M. Aouine ${ }^{\dagger}$, F.C. Santos Aires ${ }^{\dagger}$, P. Vernoux ${ }^{\dagger}$, A.S. Gay ${ }^{\ddagger}$. The Consortium Lyon St-Etienne de Microscopie (CLYM www.clym.fr) is acknowledged for access to the microscope.
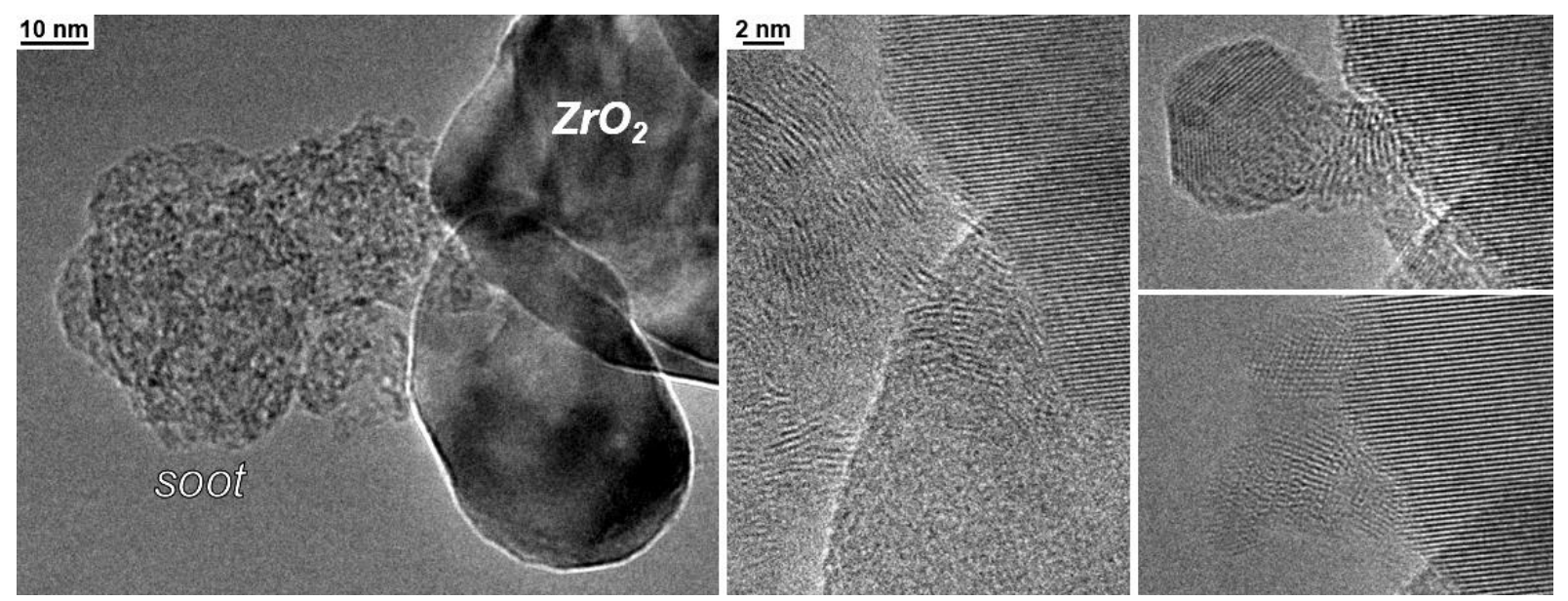

Figure 1. Combustion of soot at the contact of $\mathrm{ZrO}_{2}$ based catalysts: in situ experiment at $495^{\circ} \mathrm{C}$ under $10^{-2}$ mbar of oxygen. The total time (from left to right) is about 4 minutes. After a complete burning of the soot (right) according to the reaction $\mathrm{C}+2 \mathrm{O} \rightarrow \mathrm{CO}_{2}(\mathrm{~g}) \mathrm{Ni}$-based metallic residues crystallize as nickel oxide on the zirconia surface (ETEM $300 \mathrm{kV}$; see [3] for more details on the exact mechanism).

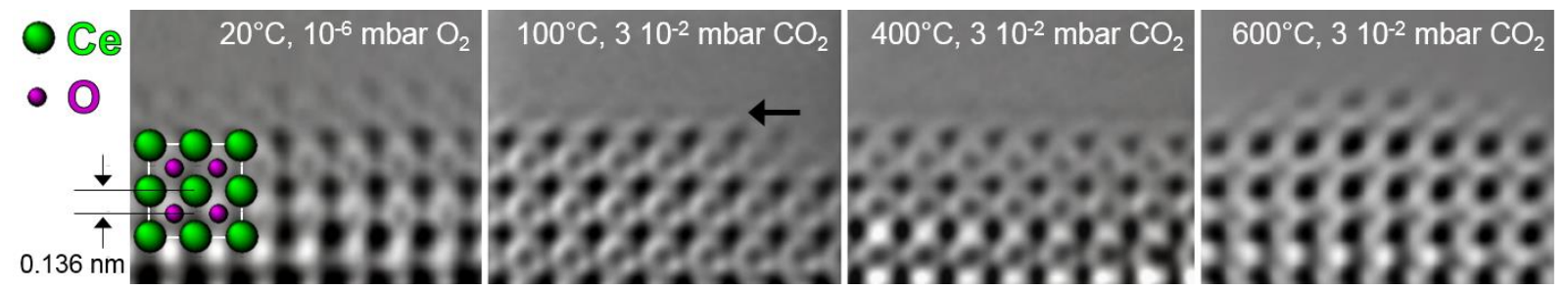

Figure 2. Tracking the atomic mobility of the (100) surface of ceria as a function of atmosphere and temperature (as indicated). All montages consist in the average of 300 aligned frames recorded at 100 fps (camera binning $2 \mathrm{Kx} 2 \mathrm{~K}$ ). Atomic columns of cerium and oxygen correspond to darker and lighter black spots respectively (with a Ce-O distance of $0.19 \mathrm{~nm}$ ). At room temperature, the 'mean' terminal plane is a Ce layer with a blurred contrast attesting a very high surface mobility. At 100 and $400^{\circ} \mathrm{C}$, the terminal plane (horizontal arrow) corresponds to the oxygen positions with a high stability but a faint contrast significantly different from the 'bulk' oxygen positions (due to absorbed carbonates). At $600^{\circ} \mathrm{C}$, the surface arrangement is comparable to that observed at room temperature, which points out the desorption of carbonates and the return to high atomic mobility. 